\title{
Cardiac surgery and healthcare quality: Is the right question being asked?
}

\author{
Saqib Masroor ${ }^{1}$ and Abdullah Nasif, MD ${ }^{1}$ \\ ${ }^{1}$ University of Toledo Medical Center
}

May 28, 2021

Title: Cardiac surgery and healthcare quality: Is the right question being asked?

Authors : Abdullah Nasif, $M D^{1} /$ Saqib Masroor, $M D^{1}{ }^{1}$ Division of Cardiothoracic Surgery, Department of Surgery, University of Toledo Medical Center Toledo, OH USA

Manuscript: Minimally Invasive Mitral Valve Surgery After Previous Sternotomy: A Propensity-Matched Analysis.Disclosure : NoneWord Count : 1381

Even though by 2003, Casselman (and many others) had concluded that totally endoscopic mitral valve repair can be performed safely with excellent results and a high degree of patient satisfaction ${ }^{1}$, less than a quarter of all isolated mitral valve procedures were performed using minimally invasive approach (MIS) by $2016^{2}$. Conventional sternotomy (ST) remains the approach of choice in the majority of cardiac surgery centers. Since 2011, partial sternotomy has fallen out of favor and right mini thoracotomy (RMT) approach has been the major MIS approach (with or without robotics) for both primary as well as re-operative mitral valve surgery. At experienced centers, the indications for MIS surgery have been expanded to include complex pathologies, reoperative surgery, endocarditis, as well as a hybrid open approach for severely calcified mitral annuli using an open deployment of transcatheter aortic valve ${ }^{3-5}$.

One reason for the slow adoption of MIS has been the lack of randomized prospective trials comparing the conventional sternotomy approach with MIS. Most literature supporting the use of MIS has consisted of retrospective review of series of individual surgeons or centers, which have shown a shorter length of stay, reduced need for transfusions and a quicker recovery ${ }^{2,3}$. Since these reports came from centers with extensive experience and the fact that initial cohorts of patients undergoing MIS were relatively lower risk patients, these retrospective observational studies were not as convincing in their conclusions, because the two groups of patients were not similar. Only a few propensity-matched analyses comparing MIS vs sternotomy have so far been reported in patients undergoing primary surgery ${ }^{4-6}$.

For re-operative mitral valve surgery, there has been one propensity-matched comparison of 42 pairs of patients undergoing right mini-thoracotomy MIS vs sternotomy from China ${ }^{7}$. MIS patients had lower transfusions, shorter length of stay and lower costs, while having similar mortality. However, the study had a mean length of stay of 22 days vs 16 days and mortality of $11 \%$ vs $7 \%$ for sternotomy and MIS patients, respectively and thus the results cannot be reliably generalized.

In this issue of the Journal, Hamandi et $\mathrm{al}^{8}$, reviewed 305 isolated MV reoperations that were performed in a single institution between 2007-2018. Patients who underwent MIS MV reoperation totaled 199, while sternotomy operations were 106 . The primary endpoints were operative mortality and 1-year survival with operative complications and length of stay being secondary endpoints. Median age of patients was 69 years with an equal gender distribution. The team performed propensity-matched analysis to compare the two groups. 
There were 88 well-balanced matched pairs. There was no statistically significant difference in mortality among the matched groups at 30 days $(3.4 \%$ vs $8.0 \%, \mathrm{p}=0.19)$ or at 1 -year $(15.9 \%$ vs. $16.5 \%, \mathrm{p}=0.9)$. Comparing long-term survival rates, no statistically significant difference was found up to 5 years postoperatively. Also, the incidence of post-operative complications such as atrial fibrillation, valve dysfunction or renal failure didn't show any statistically significant difference. However, intraoperative blood utilization was significantly lower among the MIS cohort $(\mathrm{p}<0.01)$. Patient satisfaction was not evaluated as is not possible in a retrospective analysis. Neither was readmission rates and other similar measures which would be important in a value-based care system.

The 30-day mortality difference (3.4\% vs $8 \%$ ), while not statistically significant, tended to be lower in MIS patients. 4 patients in the MIS group converted to sternotomy due to adhesions. It is not clear from the manuscript, if the mortality in the MIS group was in some way related to the conversions or not. But based on our experience over the years and from the analysis of this manuscript, we recommend an early conversion to sternotomy if one is dealing with difficult adhesions, rather than risking a long tedious operation and possibly emergently converting to sternotomy. It is also important to note that $75 \%$ of patients were discharged home, however readmission rate is unknown. With the advent of value-based purchasing, readmission rates should also be looked at. Overall, the authors should be congratulated on their excellent management of this subset of patients and for taking the time share their experience with us.

Propensity score matching is commonly used in evaluation research to estimate average treatment effects. ${ }^{9}$ The main benefit in using this statistical method is to remove confounding bias from observational cohorts. It attempts to reduce the effects of confounders by matching already treated subjects with control subjects who exhibit a similar propensity for treatment based on preexisting covariates that influence treatment selection. However, it is limited in that it requires the removal of data and works primarily on binary treatments. In this study, by including standardized mean difference (SMD), the authors were able to balance the covariates in this propensity-matched analysis.

Other than being a single-center retrospective study, this study suffered from other short-comings of a propensity match study, such as the loss of study power due to the decreased sample size after performing propensity matching. Also, "the surgeon effect" was noted. Since the MIS MV reoperative surgeries were performed by the same surgeons who performed the sternotomy cases, the results may not be generalizable.

The question being addressed by this manuscript (and by most other similar comparisons of one therapy vs another) is, "Is MIS better than sternotomy?"

Unfortunately, that question cannot be satisfactorily addressed with this or similar studies. Healthcare quality has evolved since its inception in 1999 with the Institute of Medicine report, titled "To Err is human". In the subsequent report "Crossing the Quality Chasm"11, a high-quality care is defined as beingsafe, effective, patient-centered, timely, efficient and equitable. Our healthcare delivery system is changing, and so should our research methodologies. Our analyses should go deeper than scratching the surface with mortality and morbidity data. Most studies, including this one by Hamandi et al, do not even address "effectiveness" adequately in the context of healthcare quality. Having similar mortality and morbidity means that both approaches are equally 'safe'. We have little information about other measures of safety, such as readmissions, central line associated blood borne infections. We have not evaluated whether the two approaches were patient centered (Did the patient participate in choosing the approach?), efficient (Cost of care) or equitable.

As cardiac surgeons dealing with life and death from up close, we are not used to viewing healthcare from the rather distant 6 -pronged quality viewpoint mentioned above. But this is important for a very important reason which I explain below.

Individual surgeons and patients may not have the power to bring about a meaningful change in the way we do business everyday. But just like state pension funds pressured oil companies into facing climate change ${ }^{10}$, big stakeholders like insurance companies and other payers may be able to convince the cardiac surgeons to face the future. For that to happen, quality metrics such as readmission rates, cost of care and patient satisfaction 
must be looked at and reported, because that is how these stakeholders assess quality. According to some studies $^{7}$ MIS approach is better in terms of cost and patient satisfaction. Such comprehensive analyses of quality will go a long way in answering a slightly different question than the one posed earlier; "Does MIS offer better quality than sternotomy?"

If we want to influence healthcare delivery and have a passion for quality, then our research methodology must reflect the high standards, that we have set for our clinical work. We should also develop new measures of quality besides morbidity and mortality. We have to look at those metrics that have traditionally been ignored by surgeons, but are important for the payers and the hospitals that rely on these payers for their success. As far a minimally invasive vs sternotomy approach is concerned, that question is not going to last for long. Not because one side would have won or the other lost, but because for those that have not yet boarded the train of minimally invasive mitral valve surgery, that train may have already left the station, moving at full speed ahead towards the "percutaneous station". It is not a matter of if , but when , sternotomy would not be the standard of care for mitral valve surgery. Today's vascular surgeons save open repair of abdominal aortic aneurysm for a very small subset of patients. There is no reason to believe that tomorrow's mitral valve surgeons will consider open sternotomy any differently for mitral valve surgery.

\section{References:}

1. Gammie JS, Chikwe J, Badhwar V, et al. Isolated mitral valve surgery: The Society of Thoracic Surgery adult cardiac surgery database analysis. Annals of Thoracic Surgery 2018;106(3):716-27

2. Filip P. Casselman, Sam Van Slycke, Helge Dom, Dave L. Lambrechts, Yvette Vermeulen, Hugo Vanermen, Endoscopic mitral valve repair: Feasible, reproducible, and durable, The Journal of Thoracic and Cardiovascular Surgery, Volume 125, Issue 2, 2003, Pages 273-282, ISSN 0022-5223.

3. Melnitchouk SI, Seeburger J, Kaeding AF, Misfeld M, Mohr FW, Borger MA. Barlow's mitral valve disease: results of conventional and minimally invasive repair approaches. Ann Cardiothorac Surg. 2013;2(6):768-773.

4. Lange R, Voss B, Kehl V, et al. Right minithoracotomy versus full sternotomy for mitral valve repair: A propensity matched comparison. Ann Thorac Surg 2017;103:573-9.

5. Russell HM, Guerrero ME, Salinger MH, et al. Open atrial transcatheter mitral valve replacement in patients with mitral annular calcification. J Thorac Cardiovasc Surg 2018;157:907-16.

6. Holzhey DM, Shi W, Borer MA, et al. Minimally invasive versus sternotomy approach for mitral valve surgery in patients greater than 70 years old: A propensity matched comparison. Ann Thorac Surg $2011 ; 91(2): 401-5$

7. Wang Q, Xue X, Yang J, et al. Right mini-thoracotomy approach reduces hospital stay and transfusion of mitral or tricuspid valve reoperation with non-inferior efficacy: evidence from propensity-matched study. J Thorac Dis 2018;10(8):4789-800

8. Hamandi M, Squiers JJ, Lanfear AT. Minimally invasive mitral valve surgery after previous sternotomy: A propensity-matched analysis. J Card Surg 2021.

9. Abadie, A. and Imbens, G.W. (2016), Matching on the Estimated Propensity Score. Econometrica, 84: 781-807.

10. Mooney A. How investor pressure prompted oil majors to wake up to climate change. Financial Times, August 31, 2020. https://www.ft.com/content/83b4ba6b-bef9-45d3-a6fb-087ef3143a43 Accessed May $23,2021$.

11. Institute of Medicine (U.S.). Committee on Quality of Health Care in America. Crossing the Quality Chasm: A New Health System for the 21st Century. Washington, D.C.: National Academy Press, 2001 . 